\title{
A Detailed Review and Classification Methods for ECG signal classification
}

\author{
Baljit Kaur \\ Department of Computer Science and Engineering \\ IET Bhaddal Technical Campus, Ropar, Punjab, India \\ Dr.Sanjay Singla \\ Prof. \& Head CSE \\ Department of Computer Science and Engineering \\ IET Bhaddal Technical Campus, Ropar, Punjab, India
}

\begin{abstract}
In this paper we looked into and analyzed the center and established casings of two exceptionally flawless order frameworks for ECG signal characterization. We have dissected the negatives and the positives of both of the strategies naming Multilayer recognition and Patient particular order. In the wake of breaking down every one of the realities we have finished up in backing of the better of the two subtle elements said in the later segments of this paper, in which Multilayer arrangement take an edge over patient particular strategy. This paper shows another system of grouping Arrhythmia taking into account ECG signal by utilizing Decision Tree Induction as our technique. We tried it on a standard dataset and we accomplished a normal precision of $100 \%$ utilizing our strategy.
\end{abstract}

Keywords - component; formatting; style; styling; insert (key words)

\section{INTRODUCTION}

Those tremendous measures of uses, stretching out from the customary ones for instance, such that modified character recognize and helpful examination of the extra late ones, need pulled in respectable Scrutinize exertion, for immense numbers schedules shaped and improvements submitted. Using offers for straight relationship prompts diminishment previously, trademark vector measurement, without dealing its game plan execution [1]. Distinctive researchers were Roused by the change for machines with "mind in execution that some way or another Might duplicate human execution. There were a number over-optimistic and suspicious declares made, What's more will some degree there exist strong parallels with the improvement for examination for learning based systems in the 1970s additionally neural systems in the 1980s. By inside these domains important progression need been made, particularly the spot the space covers with probability Furthermore measurements, and inside late a long while there need been numerous invigorating new advancements, both to method and procurements.

The offers recognized in the configuration recognize period need help saw as to those course of action [2]. It gives an objective pointer for right examination, determination, and solution What's more thinking ahead of the coronary sickness. Because of its crucial social worth likewise budgetary quality, it requires a broad assortment about procurements in the present day drug. That target might be to choose the people Characteristics that grant plan vectors having a spot with various Classes to have moderate What's more disjoint zones on a dimensional trademark space. The fact of the matter is twofold: decreasing those enter qualities for MLP along these lines correspondingly as on less demanding count load, Also Comprehension which beat Characteristics accept basic parts in recognizing more than four ecg outlines [3]. The feasibility of the representational space (highlight set) might be determined toward how extraordinary case from unmistakable classes' cam wood is isolated. Accommodated a set from guaranteeing readiness outlines from every class, that destination might be will secure decision limits in the trademark space which separate plans having a spot ought to particular classes. In the quantifiable decision theoretic methodology, those decision points of confinement are determined by those probability disseminations of the illustrations having a spot ought to each class, which should perhaps be indicated or insightful. For the outline recognize unit, reinforce ahead and totally related mimicked neural systems, which would preferably wanted to each tolerant toward those proposed multidimensional particle swarm streamlining method, would used [4]. It is could be 
permitted that those available information will be expansion for A quick result However might be insufflate for grasping a more noteworthy sum general widely appealing issue.

The Taking in method incorporates redesigning framework basic building in addition affiliation weights something to that effect that a framework may adequately play out a specific characterization/bunching errand. Truthful components were concentrated beginning with the heart rate variability (HRV) and the ecg signs What's more were used to illustration recognize Throughout those particular menstrual periods [5]. The extending Ubiquity from guaranteeing neural framework models with deal with outline recognize issues need been crucial due to their obviously low dependence once space particular adapting Furthermore as a result of the openness from asserting powerful Taking in estimations to experts to use.

Design acknowledgment as a field of study grew essentially in the 1960s. It was particularly an interdisciplinary subject, covering improvements in the regions of measurements, building, manmade brainpower, software engineering, brain science and physiology, among others. Some individuals entered the field with a genuine issue to unravel. The extensive quantities of uses, running from the traditional ones, for example, programmed character acknowledgment and therapeutic conclusion to the later ones, have pulled in significant exploration exertion, with numerous strategies created and progresses made. Utilizing highlights with direct relationship prompts lessening in highlight vector measurement, without trading off its characterization execution [8]. Other analysts were spurred by the improvement of machines with "brain like execution that somehow could imitate human execution. There were numerous over-hopeful and unreasonable cases made, and to some degree there exist solid parallels with the development of exploration on information based frameworks in the 1970s and neural systems in the 1980s. In any case, inside these zones huge advancement has been made, especially where the area covers with likelihood and insights, and inside late years there have been numerous energizing new improvements, both in philosophy and applications. These expand on the strong establishments of prior examination and exploit expanded computational assets promptly accessible these days.

Since 1903 the ECG was acquainted with clinical pharmaceutical, the strategies have been created quickly in the record, preparing and determination of the ECG whether it is in the biomedical region or in designing and we gather significant experience.ECG assumes a critical part in the clinical finding of the coronary illness. The components identified in the example acknowledgment stage are considered for the order [9]. It gives a target pointer to right investigation, analysis, treatment and consideration of the coronary illness. Due to its vital social quality and monetary worth, it has an extensive variety of utilizations in the cutting edge prescription. ECG is still a noteworthy exploration subject in the biomedical designing. Numerous examination and work on breaking down ECG flags, for example, those by Conde (1994) and van Bemmel (1982) have been accounted for as of late . Recognition of genuine crest effectively is completely key as it showed the state of heart and additionally reflects to its usefulness.

In the factual methodology, every example is spoken to regarding $\mathrm{d}$ elements or estimations and is seen as a point in a d-dimensional space. The objective is to pick those components that permit design vectors having a place with various classes to involve minimal and disjoint areas in a dimensional element space. The point is twofold: lessening the info qualities of MLP to lower calculation weight, and understanding which pulse highlights assume imperative parts in perceiving above four ECG designs [10]. The viability of the representation space (highlight set) is dictated by how well examples from various classes can be isolated. Given an arrangement of preparing examples from every class, the goal is to build up choice limits in the component space which separate examples having a place with various classes. In the measurable choice theoretic methodology, the choice limits are dictated by the likelihood conveyances of the examples having a place with every class, which should either be indicated or learned .One can likewise take a discriminates investigation based way to deal with order: First a parametric type of the choice limit (e.g., straight or quadratic) is determined; then the "best" choice limit of the predefined structure is discovered in light of the characterization of preparing examples. Such limits can be built utilizing, for instance, a mean squared mistake standard. The immediate limit development methodologies are upheld by Vapnik's theory: "On the off chance that you have a confined measure of data for taking care of some issue, attempt to take care of the issue specifically and never tackle a more broad issue as a middle stride. For the example acknowledgment unit, feed forward and completely associated simulated neural systems, which are ideally intended for every patient by the proposed multidimensional molecule swarm streamlining procedure, are utilized [4]. It is conceivable that the accessible data is adequate for an immediate arrangement yet is lacking for taking care of a more broad middle issue." 


\section{LITERATURE REVIEW LITERATURE REVIEWS}

Sheha et.al in [1] Electrocardiogram (ECG) holds quick and dirty dominant part of the information as to unplanned irregularity of an at risk. Manual Investigation for a long time ecg record will be A long change. Electronic ecg Investigation backs clinicians in decision making. Same time sketching out A negligible exertion symptomatic help framework, objectives on the structure resources cutoff those get ready velocity, unavoidably impacting those steadfast quality.

Jafari et.al to [2] this paper, tended to those issue about allocating a perplexing calculation same time the Vitality use due to remote transmission is minimized. Ecg Investigation figuring's routinely contained from guaranteeing preprocessing, case acknowledgment, Furthermore plan. Perceiving the presentation of the ecg drives, they created A technique ought to perform preprocessing and outline recognition by and large done little embedded systems associated of the heads. Those Characteristics recognized in the outline recognition period need help recognized to the game plan. Preferably, assuming that the elements recognized to every heartbeat live for a lone get ready hub, the transmission will be superfluous.

Lin et.al in [3] paper displayed a methodology In light of the mixing of multilayer perceptions (MLP) Furthermore plan tree (CT) to perceiving four electrocardiograms (ECG) designs: ordinary, got out pack expansion piece (LBBB), straight pack appendage piece (RBBB) and untimely ventricular withdrawal (PVC). This examination uses MIT/BIH arrhythmia database as get ready and attempting data.

Ince et.al in [4] paper presented a nonexclusive What's more patient-particular plan structure plot to generous Furthermore correct recognizable proof of ecg heartbeat outlines. The suggested trademark extraction philosophy utilizes morphologic wavelet change highlights, which need help foreseen onto a less demanding dimensional trademark space using focal part investigation, What's more transient Characteristics from those ECG data. To those configuration recognize unit, reinforce forward and totally related mimicked neural systems, which need help in a perfect world sketched out to each tolerant toward the recommended multidimensional atom swarm streamlining method, would used.

Champaty et.al in [5], attempts were bothered ought to organize menstrual stages from asserting junior sound female (21-25 years) In light of the offers obtained beginning with ecg signs. Quantifiable offers were concentrated from the heart rate variability (HRV) and the ecg signs Furthermore were used for instance recognition Throughout the unmistakable menstrual periods.

Agrafioti et.al in [6] this value of exertion brought of the table the ECG sign and shows a cautious examination of its mental properties. Those way that this pointer need been made concerning delineation a biometric trademark calls for subject-subordinate feeling recognizers that catch the quick variability of the marker beginning with its homeostatic benchmark. An answer In light of those exploratory mode rot is prescribed to the distinguishing proof of quickly developing feeling outlines as for ECG. Course of action offers need help In light of the quick repeat (HilbertHuang change) and the area influencing inside every mode. Two test setups need help showed to the elicitation for element excitement and uninterested excitement/valence.

Tuzcu et.al in [7] they presented a novel system for the request and ID number of electrocardiograms (ECGs) of Different heart beat unsettling influences. This might be a key endeavor in those modified analyzation about heart beat unsettling influences. Changing event when twisting (DTW) will be used thus. DTW might be utilized adequately for talk recognition. Wavelet examination is used inside a part implantable cardioverter defibrillators at this moment to ECG waveform recognition Also arrange inspiration. The reenactments about time-arrangement ecg data about Different beat aggravations need help took care of. Run of the mill sinus rhythm ecg formats are stood out from the imitated rhythms Eventually Tom's examining both systems.

Sheha et.al in [8] outlined a minimal effort indicative emotionally supportive network; imperatives on the framework assets constrain the handling speed, in the end influencing the unwavering quality. To determine these issues, three key components have been tended to in this study: the element extraction strategy, all out number of elements and the database utilized. For highlight extraction, 'polar Teager vitality' calculation has been created, yielding about $70 \%$ sparing in preparing time when contrasted with other understood techniques. Utilizing highlights with straight relationship prompts lessening in highlight vector measurement, without bargaining its grouping execution. Hence the straight relationship between two ECG highlights, in particular 'educational entropy'(S) and 'mean Teager vitality' has been uncovered. These elements are used for ECG beat order utilizing 'fluffy C-implies grouping' calculation. The calculation is assessed utilizing the MIT-BIH database and after that tried by ECG measured with the cardio-care unit. The QRS recognition execution of the proposed technique is great, with $0.27 \%$ identification mistake rate. For order of ECG beats, normal affectability and positive expectation rate accomplished are $98.93 \%$ each. 
Jafari et.al in [9] tended to the issue of parceling such an intricate calculation while the vitality utilization because of remote transmission is minimized. ECG examination calculations typically comprise of preprocessing, example acknowledgment, and order. Considering the introduction of the ECG drives, they formulated a strategy to perform preprocessing and design acknowledgment locally in little implanted frameworks appended to the leads. The elements recognized in the example acknowledgment stage are considered for the characterization. In a perfect world, if the elements distinguished for every pulse live in a solitary preparing hub, the transmission will be superfluous. Something else, to perform arrangement, the elements must be assembled on a neighborhood hub and, along these lines, the correspondence is inescapable. They perform such an element gathering by displaying the issue as a hyper graph and applying dividing plans which yield a noteworthy force sparing in remote correspondences. Besides, they used element reconfiguration by programming module relocation. This strategy, as for dividing, upgrades the general force sparing in such frameworks. Also, it adaptively adjusts the framework arrangement in different situations and on various patients. They assessed the viability of their proposed procedures on MIT/BIH benchmarks and, by and large, accomplish 70 percent vitality sparing.

Lin et.al in [10] exhibited a methodology taking into account the mix of multilayer perceptions (MLP) and grouping tree (CT) to perceiving four electrocardiograms (ECG) designs: ordinary, left package branch square (LBBB), right package branch piece (RBBB) and untimely ventricular withdrawal (PVC). This study uses MIT/BIH arrhythmia database as preparing and testing information. They initially connected MLP and CT individually to perceive ECG designs. Next, they gathered the ECG signal elements which are chosen in part CT's hub, and food these chose highlights into MLP for ECG design acknowledgment. The point is twofold: lessening the information qualities of MLP in order to lower calculation weight, and understanding which pulse highlights assume essential parts in perceiving above four ECG designs. To look at the viability of proposed strategy, they considered the important segment investigation (PCA) that was much of the time used to chop down the information measurement for example acknowledgment. Far reaching PC recreations will legitimize the possibility of the proposed approach.

Ince et.al in [11] introduced a non specific and patient-particular order framework intended for powerful and precise recognition of ECG pulse designs. The proposed highlight extraction process uses morphological wavelet change highlights, which are anticipated onto a lower dimensional element space utilizing foremost part examination, and worldly elements from the ECG information. For the example acknowledgment unit, feed forward and completely associated simulated neural systems, which are ideally intended for every patient by the proposed multidimensional molecule swarm enhancement strategy, are utilized. By utilizing generally little regular and patientparticular preparing information, the proposed arrangement framework can adjust to huge interpatient varieties in ECG designs via preparing the ideal system structure, and in this manner, accomplishes higher precision over bigger datasets. The characterization tests over a benchmark database exhibit that the proposed framework accomplishes such normal correctness's and sensitivities superior to anything the greater part of the present best in class calculations for identification of ventricular ectopic beats (VEBs) and supra-VEBs (SVEBs). Over the whole database, the normal precision affectability exhibitions of the proposed framework for VEB and SVEB recognitions are $98.3 \%-84.6 \%$ and $97.4 \%-63.5 \%$, separately. At long last, because of its parameter-invariant nature, the proposed framework is exceptionally non specific, and along these lines, pertinent to any ECG dataset.

Champaty et.al in [12] characterized menstrual periods of youthful solid female (21-25 years) in view of the components acquired from ECG signals. Factual components were separated from the heart rate variability (HRV) and the ECG flags and were utilized for example acknowledgment amid the distinctive menstrual stages. The example acknowledgment ponders utilizing HRV highlights proposed that the menstrual stage grouping proficiency were $>85$ $\%$ and $>90 \%$ utilizing Multilayer perception (MLP) and Radial premise capacity system (RBF) Artificial Neural Network (ANN) models. Then again, the example acknowledgment concentrates on utilizing ECG signal components indicated arrangement efficiencies of $>80 \%$ and $>90 \%$ utilizing MLP and RBF ANN models. The outcomes showed interim changes in the autonomic sensory system and the heart physiology of the volunteers amid the menstrual cycle.

Agrafioti et.al in [13] exhibited an intensive examination of its mental properties. The way that this sign has been built up as a biometric trademark calls for subject-subordinate feeling recognizers that catch the momentary variability of the sign from its homeostatic pattern. An answer in light of the experimental mode disintegration is proposed for the location of powerfully developing feeling designs on ECG. Characterization components depend on the immediate recurrence (Hilbert-Huang change) and the neighborhood swaying inside each mode. Two test setups are displayed for the elicitation of dynamic excitement and uninvolved excitement/valence. The outcomes bolster the desires for subject specificity, and also exhibiting the attainability of deciding valence out of the ECG morphology (up to 89 percent for 44 subjects). Likewise, this work separates surprisingly amongst dynamic and uninvolved excitement and promoters that there are higher odds of ECG reactivity to feeling when the instigation technique is dynamic for the subject. 
Tuzcu et.al in [14] introduced a novel technique for the grouping and distinguishing proof of electrocardiograms (ECGs) of different heart beat unsettling influences. This is a vital stride in the programmed investigation of heart beat unsettling influences. Dynamic time twisting (DTW) is utilized for this reason. DTW is used effectively in discourse acknowledgment. Wavelet investigation is utilized as a part of some implantable cardioverter defibrillators as of now for ECG waveform acknowledgment and order reason. The recreations of time-arrangement ECG information of different mood unsettling influences are created. Typical sinus musicality ECG layouts are contrasted with the mimicked rhythms by both techniques. DTW investigation effectively separates the ECGs of different arrhythmias. Of note, DTW can separate ventricular tachycardia from supraventricular tachycardia not at all like wavelet investigation. Separation of these two cadence sorts has huge clinical ramifications. DTW can possibly be utilized for programmed design acknowledgment of ECG changes illustrative of different cadence unsettling influences.

\section{AUTOMATED PATIENT SPECIFIC CLASSIFICATION}

In this examination the creators have proposed an exceptionally viable yet now primitive technique for arrangement. They have utilized the main food forward neural systems which bargain with the criticality of the exploration. This exploration has used a generally little information set and they themselves have conceded this in the earliest reference point of the paper. Having extensive information set which is at force with multifaceted nature of the work is imperative. They have additionally utilized patient particular information set which utilizes a little additionally extremely restricted variety decreasing the heartiness of the order. We can utilize just the general information set to defeat the issue of strength in the arrangement of ECG signs. We can utilize the extensive information set in light of the fact that our emphasis is on expanding the exactness as opposed to diminishing the computational burden. We can guarantee the variability of the element to enhance the preparation and testing procedures of our framework.

\section{Multilayer Perceptions AND ClassificAtion tREe}

In this examination the makers have proposed an especially feasible yet now primitive method for course of action. They have used the primary nourishment forward neural frameworks which deal with the criticality of the investigation. This investigation has utilized a by and large little data set and they themselves have yielded this in the most punctual reference purpose of the paper. Having a broad data set which is at power with multifaceted nature of the work is basic. They have moreover used a patient specific data set which uses a little furthermore to a great degree confined assortment diminishing the generosity of the request. We can use only the general data set to vanquish the issue of quality in the game plan of ECG signs. We can use the broad data set in light of the way that our accentuation is on growing the precision instead of lessening the computational weight. We can promise the variability of the component to upgrade the planning and testing strategies of our system.

\section{PROBLEM FORMULATION}

An electrocardiogram (ECG) is an electrical recording of the heart that outlines the cardiovascular cycle. It is routinely used as a first course of choice in diagnosing various cardiovascular contaminations. In any case, while most existing work has tended to the issue of separating the ECG of a lone patient, we focus on the issue of filtering for planning ECGs from a database. Along these lines, it is the essential valuable utilization of substance based recuperation procedures for conclusion endorsement in the midst of decision sponsorship. Next, our structure considers both sifted and modernized ECGs. Finally, our ECG waveform digitization figuring is totally modified and does not require overseer intercession to set structure parameters. Yet most centers now have automated ECG recorders, a noteworthy part of the legacy ECG data is still in paper structure, out of range to examination by PC. Opening these ECG records engraved on paper and showing them to cutting edge examination would be significant. It gives additional data to current ECG examination methodology, and what's more chronicled data to be used for close studies. For example, the Framingham Heart Study has been social event ECG data since 1948, with the data accumulated before 1990 in printed rendition structure. Quantifiable or look for based ECG examination methodology that require generous ECG databases can be populated with such examples of legacy data. Thusly the examination of paper ECG may at present be material for disease specific recuperation. The components assume a critical part in the discovery of examples in the ECG investigation. To have an adequately enormous list of capabilities is vital. The present study does not utilize the required size of list of capabilities. Automated ECG examination reinforces clinicians in decision making. While plotting an insignificant exertion symptomatic candidly steady system, prerequisites on the structure resources limit the planning speed, at last affecting the relentless quality. To decide these issues, three key components have been tended to in this study: the component extraction procedure, hard and fast number of components and the database used. For highlight extraction, 'polar Teager essentialness' computation has been made, yielding very nearly $70 \%$ saving in taking care of time when stood out from other doubtlessly 
comprehended techniques. Using highlights with straight relationship prompts diminishment in highlight vector estimation, without bartering its plan execution. Thus the immediate relationship between two ECG highlights, specifically 'instructive entropy'(S) and 'mean Teager essentialness' has been revealed. These segments are utilized for ECG beat gathering using 'cushy C-suggests packing' figuring. The estimation is surveyed using the MIT-BIH database and after that attempted by ECG measured with the cardio-care unit. The QRS distinguishing proof execution of the proposed technique is extraordinary, with $0.27 \%$ acknowledgment goof rate. For plan of ECG beats, ordinary affectability and positive desire rate finished are $98.93 \%$ each. The little list of capabilities causes the issue of non solid precision furthermore decreased exactness. We propose an upgraded highlight set choice and utilizing a classifier which can possibly build the precision with a nice estimated highlight set. We have choose to utilize choice tree affectation as our classifier.

\section{PROPOSED WORK}

In the present work, we have developed an ECG pattern detection technique using Decision Tree Induction (DTI).

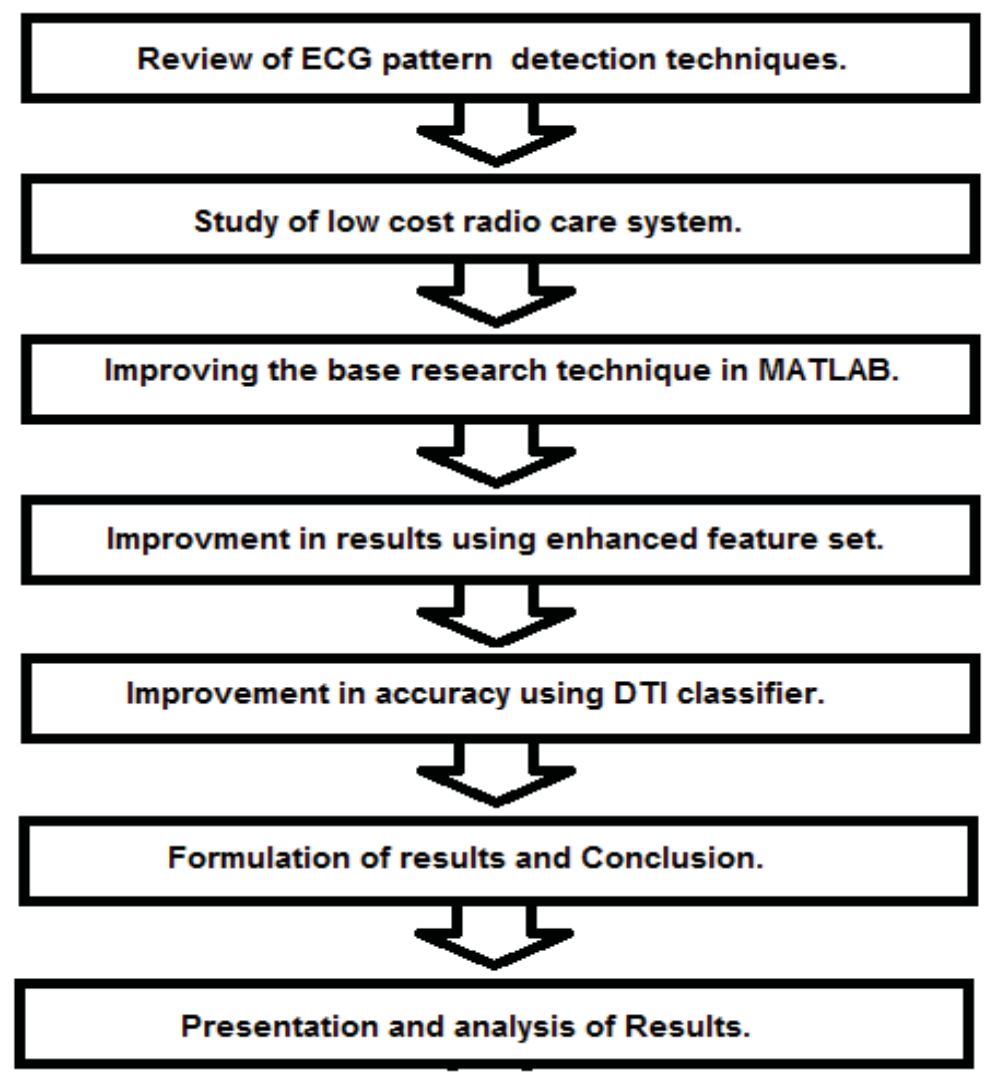

Figure 1: Proposed Methodology

Above all else we took a dataset of Arrhythmia. With this dataset we connected component area strategy to choose essential elements. We utilized Principle Component Analysis for the same. After that we partitioned the information into preparing and testing set. At that point we firstly connected Fuzzy C-mean bunching and afterward Decision Tree Induction on the same dataset and afterward tried the outcomes. The outcome utilizing DTI was $100 \%$ exactness. 


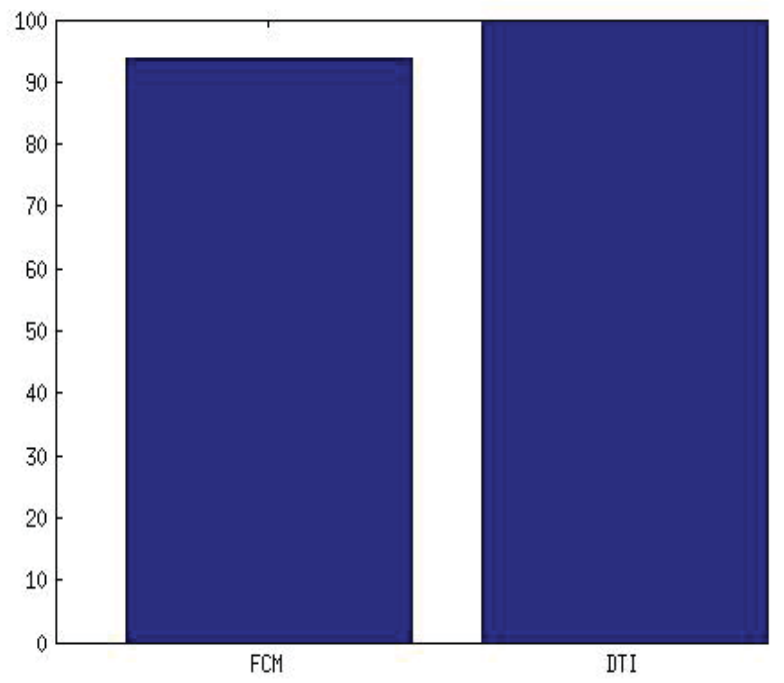

Figure 2: Accuracy of both the Systems (FCM and DTI). DTI has 100\% accuracy.

The steps are as follow in the flowchart:

Taking into account the flowchart we can see the strides unmistakably. We have executed the test code in MATLAB and utilized UCI library for the dataset of Arrhythmia. The strides are as tail, we stacked the dataset in MATLAB. We isolated the Dataset into Training and Testing Sets. We connected Principal Component Analysis on the dataset to lessen the dimensionality of it.

We then utilized the preparation piece of it to prepare a Decision Tree Classifier utilizing Supervised Learning Approach and a Fuzzy C-mean Object utilizing unsupervised methodology.

$$
c_{k}=\frac{\sum_{x} w_{k}(x)^{m} x}{\sum_{x} w_{k}(x)^{m}} .
$$

We then tested the results of both the techniques and presented in form of bar graph.

\section{I. CONCLUSION}

In this survey we have thought about two technique utilized for the grouping of ECG signs. Both the strategies looked into have their own favorable circumstances and hindrances however the Multilayer arrangement guarantees more exactness in light of the fact that the framework truly tests the information conveniently. Then again the patient particular characterization depends entirely upon the little patient particular database which truly bargains with the situational capability of the exploration. Finishing up, we would say that the Multilayer grouping with order trees is a greatly improved methodology as far as characterization of ECG signs. In this paper, we have proposed a technique for recognizing ECG flags and characterize Arrhythmia utilizing Decision Tree Induction. Our outcomes are promising and showcases a $100 \%$ exactness on our dataset which was taken from UCI library. 


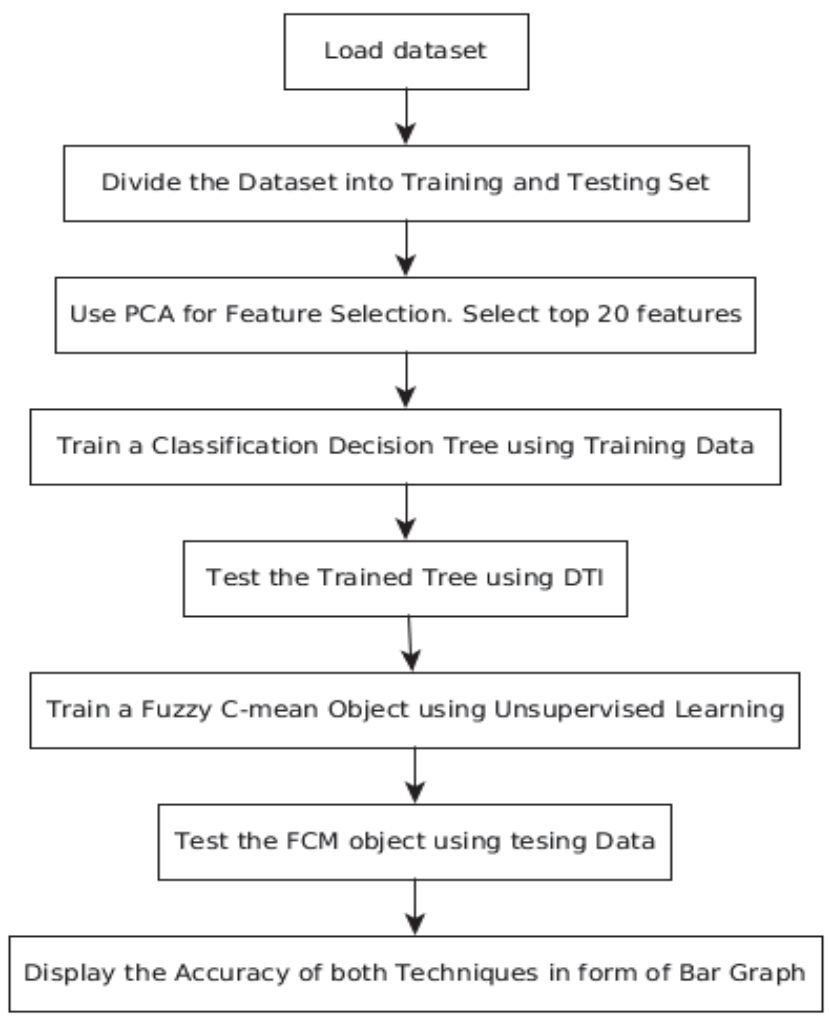

Figure 3: Flowchart of the System

\section{REFERENCES}

[1] Sheha, Mariam Ahmed, Amr Sharwy, and Mai S. Mabrouk. "Pigmented skin lesion diagnosis using geometric and chromatic features." Biomedical Engineering Conference (CIBEC), 2014 Cairo International. IEEE, 2014.

[2] Jafari, R., Noshadi, H., Ghiasi, S. and Sarrafzadeh, M. Adaptive electrocardiogram feature extraction on distributed embedded systems.Parallel and Distributed Systems, IEEE Transactions on, 17(8), pp.797-807, 2016.

[3] Lin, Y.J., Tsai, S.N. and Yang, J.X., May. Learning ECG Patterns with the Aid of Multilayer Perceptrons and Classification Trees. In Bioinformatics and Biomedical Engineering, 2008. ICBBE 2008. The 2nd International Conference on (pp. 1859-1862). IEEE, 2008.

[4] Ince, T., Kiranyaz, S. and Gabbouj, M. A generic and robust system for automated patient-specific classification of ECG signals. Biomedical Engineering, IEEE Transactions on, 56(5), pp.1415-1426, 2009.

[5] Champaty, B., Bhandari, S., Pal, K. and Tibarewala, D.N., December. Artificial intelligence based classification of menstrual phases in amenorrheic young females from ECG signals. In India conference (INDICON), 2013 annual IEEE (pp. 1-6). IEEE, 2013.

[6] Agrafioti, F., Hatzinakos, D. and Anderson, A.K.. ECG pattern analysis for emotion detection. Affective Computing, IEEE Transactions on,3(1), pp.102-115, 2012.

[7] Tuzcu, V. and Nas, S. October. Dynamic time warping as a novel tool in pattern recognition of ECG changes in heart rhythm disturbances. InSystems, Man and Cybernetics, 2005 IEEE International Conference on(Vol. 1, pp. 182-186). IEEE, 2005.

[8] Shorten, G.P. and Burke, M.J.August. A time domain based classifier for ECG pattern recognition. In Engineering in Medicine and Biology Society, EMBC, 2011 Annual International Conference of the IEEE(pp. 4980-4983). IEEE, 2011.

[9] Gupta, A., Thomas, B., Kumar, P., Kumar, S. and Kumar, Y., September. Neural Network based indicative ECG classification. InConfluence The Next Generation Information Technology Summit (Confluence), 2014 5th International Conference- (pp. 277-279). IEEE, 2014.

[10] Gomes, P.R., Lima, C.S., Soares, F.O. and Correia, J.H., 2011, August. Automatic continuous ECG monitoring system for over-drug detection in Brugada Syndrome. In Engineering in Medicine and Biology Society, EMBC, 2011 Annual International Conference of the IEEE (pp. 7928-7931). IEEE, 2011. 